toc "contentsline section "numberline 1.A construction of formal Frobenius manifolds18 toc"contentsline subsection "numberline 1.1.Frobenius algebras and formal Frobenius manifolds18 toc"contentsline subsection"numberline 1.2.DGBV algebras19 toc "contentsline subsection "numberline 1.3.Integral on DGBV algebras19 toc "contentsline subsection "numberline 1.4.Formal Frobenius supermanifolds from DGBV algebras20 toc "contentsline subsection "numberline 1.5.Potential function20 toc "contentsline section "numberline 2.Formal Frobenius manifold structure on de Rham cohomology21 toc"contentsline subsection "numberline 2.1.DGBV algebra in Poisson geometry21 toc "contentsline】 subsection "numberline 2.2.De Rham Frobenius manifolds for Kähler manifolds24 toc "contentsline subsection "numberline 2.3.Explicit formula for $\Gamma 25$ toc "contentsline section "numberline 3.Identification with formal Frobenius supermanifold on Dolbeault cohomology26 toc "contentsline subsection "numberline 3.1.Formal Frobenius manifold structure on Dolbeault cohomology27 toc "contentsline subsection "numberline 3.2.The identification27 toc"contentsline section "numberline 4.Acknowledgements28 toc"contentsline sectionReferences 28

Mathematical Research Letters 6, 17-29 (1999)

\title{
IDENTIFICATION OF TWO FROBENIUS MANIFOLDS
}

\author{
Huai-Dong CaO and Jian Zhou
}

\begin{abstract}
We identify two Frobenius manifolds obtained from two different differential Gerstenhaber-Batalin-Vilkovisky algebras on a compact Kähler manifold. One is constructed on the Dolbeault cohomology in Cao-Zhou [6], and the other on the de Rham cohomology in the present paper. This can be considered as a generalization of the identification of the Dolbeault cohomology ring with the complexified de Rham cohomology ring on a Kähler manifold.
\end{abstract}

One of the far-reaching ideas from string theory is that of mirror symmetry [30]. On a given Calabi-Yau manifold $M$, string theory suggests two kinds of super conformal field theories: the $A$ theory and the $B$ theory. The mirror symmetry suggests the existence of another Calabi-Yau manifold $\widehat{M}$, called a mirror manifold of $M$, such that the $A$ theory on $M$ can be identified with the $B$ theory on $\widehat{M}$, and vice versa. The constructions of mirror manifolds of quintics in $\mathbb{C P}_{4}$ were given by Greene and Plesser [10]. Candelas et al used their constructions to conjecture a formula [5] on the number of rational curves of any degree on a quintic in $\mathbb{C P}_{4}$. Recently, Lian-Liu-Yau [15] have proposed and studied the important Mirror Principle. Among its many applications, a proof of the formula of Candelas et al was given, completing the program of Candelas et al, Kontsevich, Manin and Givental.

A closely related idea from string theory is that of quantum cohomology. We will not review this rapidly progressing theory. One way to formulate quantum

Received June 23, 1998.

Both authors were supported in part by NSF. 
cohomology is via the notion of Frobenius manifold introduced and extensively studied by Dubrovin [7, 8]. Now a version of the Mirror Symmetry Conjecture can be formulated as the identification of Frobenius manifold structures obtained by different constructions. (For an exposition of this point of view, the reader is referred to a recent paper by Manin [17].) More precisely, on a Calabi-Yau manifold $X$, there are two natural algebras

$$
A(X)=\oplus_{p, q} H^{q}\left(X, \Omega^{p}\right), \quad B(X)=\oplus_{p, q} H^{q}\left(X, \Omega^{-p}\right),
$$

where $\Omega^{-p}$ is the sheaf of holomorphic sections to $\Lambda^{p} T X$. By Hodge theory, $A(X)$ is isomorphic to the de Rham cohomology with complex coefficients $H_{d R}^{*}(X, \mathbb{C})$. There is a construction of Frobenius manifold structure on the de Rham cohomology given by the theory of quantum cohomology. For example, see Ruan and Tian's work [21] on the mathematical formulation of quantum cohomology. By Bogomolov-Tian-Todorov theorem, the moduli space of complex structures on $X$ is an open subset in $H^{1}\left(X, \Omega^{-1}\right)$. Witten [27] suggested the construction of an extended moduli space of complex structures. In Ran [20], this problem was studied and differential Gerstenhaber-Batalin-Vilkovisky (DGBV) algebra structure on $\Omega^{-*, *}(M)$ was found via Koszul's work [13]. Note that Gerstenhaber algebra structure on $H^{-*, *}(M)$ was observed in Gerstenhaber-Schack [9], $\S 27$. For Calabi-Yau manifolds, the work of Bershadsky-Cecotti-Ooguri-Vafa [2] (Kodaira-Spencer theory of gravity) is very important (especially §5). Based on the above works, Barannikov and Kontsevich [1] gave a construction of a formal Frobenius manifold structure on $B(X)$. They also remarked that the construction of Frobenius manifold structure can be carried out for general DGBV algebras with some suitable conditions. The details have been given in Manin [17]. Notice that the above constructions of Frobenius manifold structures on $A(X)$ and $B(X)$ are of totally different nature. Motivated by mirror symmetry, it is natural to seek for a construction of formal Frobenius manifold structure on $A(X)$ via DGBV algebra approach. Such a construction was given by the authors in [6]. It is interesting to compare our work [6] with the theory of Kähler gravity of Bershadsky and Sadov [3].

For a Calabi-Yau manifold $M$, if $\widehat{M}$ is a proposed mirror manifold (constructed along the lines of Greene-Plesser [10] or Strominger-Yau-Zaslow [22]), then it is natural to conjecture that the formal Frobenius manifold structure on $B(M)$ constructed by Barannikov-Kontsevich [1] can be identified with that on $A(\widehat{M})$ constructed by the authors [6]. Therefore it is very important to study the problem of when two DGBV algebras give rise to identical formal Frobenius manifold structures on their cohomology. In this paper, we give a result in this direction. First, it is well-known that there is a DGBV algebra structure on $\Omega^{*}(X)$ for any Poisson manifold $X$. When $X$ is closed and Kähler, we show that the conditions in Manin [17] are satisfied, hence give a construction of a formal Frobenius manifold structure on $H_{d R}^{*}(X, \mathbb{C})$ via DGBV algebra approach. Our main result is that this formal Frobenius manifold structure can be identified with the one on $A(X)$ constructed in [6]. 


\section{A construction of formal Frobenius manifolds}

In this section, we review a construction of formal Frobenius supermanifolds. For details, the reader should consult the papers by Tian [23], Todorov [24], Barannikov-Kontsevich [1] and Manin [17]. Here, we follow the formulation in Manin [17].

1.1. Frobenius algebras and formal Frobenius manifolds. A commutative associatve algebra $(H, \cdot)$ with unit 1 over a field $k$ is called a Frobenius algebra if there is a symmetric nondegenerate inner product $(\cdot, \cdot)$ such that

$$
(a \cdot b, c)=(a, b \cdot c),
$$

for any $a, b, c \in H$. Take a basis $\left\{e_{a}\right\}$ of $H$ such that $e_{0}=1$. Let $\eta_{a b}=\left(e_{a}, e_{b}\right)$ and $\left(\eta^{a b}\right)$ be the inverse matrix of $\left(\eta_{a b}\right)$. Denote by $\left\{x^{a}\right\}$ the linear coordinates in the basis $\left\{e_{a}\right\}$. For our purpose, a formal Frobenius manifold structure on $H$ is a formal power series $\Phi$ in $x^{a}$ 's, such that

$$
\left(\frac{\partial^{3} \Phi}{\partial x^{0} \partial x^{a} \partial x^{b}}\right)=\left(\eta_{a b}\right)
$$

and $\Phi$ satisfies the WDVV equations:

$$
\frac{\partial^{3} \Phi}{\partial x^{a} \partial x^{b} \partial x^{p}} \eta^{p q} \frac{\partial^{3} \Phi}{\partial x^{q} \partial x^{c} \partial x^{d}}=\frac{\partial^{3} \Phi}{\partial x^{b} \partial x^{c} \partial x^{p}} \eta^{p q} \frac{\partial^{3} \Phi}{\partial x^{a} \partial x^{q} \partial x^{d}} .
$$

The formal power series $\Phi$ is called the potential function. It is straightforward to extend the above definition to the $\mathbb{Z}_{2}$-graded version. For general definition of Frobenius manifolds, see Dubrovin [8] or Manin [16].

1.2. DGBV algebras. A $\mathbb{Z}_{2}$-graded Gerstenhaber algebra consists of a triple $\left(\mathcal{A}=\oplus_{i \in \mathbb{Z}_{2}} \mathcal{A}^{i}, \wedge,[\cdot \bullet \cdot]\right)$, such that $(\mathcal{A}, \wedge)$ is a $\mathbb{Z}_{2}$-graded commutative associative algebra over a filed $k$, and

$$
\begin{gathered}
{[a \bullet b]=-(-1)^{(|a|+1)(|b|+1)}[b \bullet a],} \\
{[a \bullet[b \bullet c]]=[[a \bullet b] \bullet c]+(-1)^{(|a|+1)(|b|+1)}[b \bullet[a \bullet c]],} \\
{[a \bullet(b \wedge c)]=[a \bullet b] \wedge c+(-1)^{(|a|+1)|b|} b \wedge[a \bullet c],}
\end{gathered}
$$

for all homogeneous $a, b, c \in \mathcal{A}$. Let $\Delta$ be a linear operator of odd degree such that $\Delta 1=0$, we say $\Delta$ generates the Gerstenhaber bracket if $[\cdot \bullet \cdot]_{\Delta}=[\cdot \bullet \cdot]$, where

$$
[a \bullet b]_{\Delta}=(-1)^{|a|}\left(\Delta(a \wedge b)-\Delta a \wedge b-(-1)^{|a|} a \wedge \Delta b\right),
$$

for all homogeneous $a, b \in \mathcal{A}$. In this case, the tuple $(\mathcal{A}, \wedge,[\cdot \bullet \cdot], \Delta)$ is called a Gerstenhaber-Batalin-Vilkovisky algebra (GBV algebra). A DGBV (differential Gerstenhaber-Batalin-Vilkovisky) algebra is a GBV algebra with a $k$-linear derivation $\delta$ of odd degree with respect to $\wedge$, such that

$$
\delta^{2}=\delta \Delta+\Delta \delta=0 .
$$

We will be interested in the cohomology group $H(\mathcal{A}, \delta)$.

The examples of DGBV algebras are abundant in differential geometry. See, for example, Koszul [13] and Xu [28] in Poisson geometry, Tian [23], Ran [20] 
and Barannikov-Kontsevich [1] in deformation theory, Cao-Zhou [6] in Kähler geometry.

1.3. Integral on DGBV algebras. A $k$-linear functional $\int: \mathcal{A} \rightarrow k$ on a DGBV-algebra is called an integral if for all $a, b \in \mathcal{A}$,

$$
\begin{aligned}
& \int(\delta a) \wedge b=(-1)^{|a|+1} \int a \wedge \delta b, \\
& \int(\Delta a) \wedge b=(-1)^{|a|} \int a \wedge \Delta b .
\end{aligned}
$$

Under these conditions, it is clear that $\int$ induces a scalar product on $H=$ $H(\mathcal{A}, \delta):(a, b)=\int a \wedge b$. If it is nondegenerate on $H$, we say that the integral is nice. It is obvious that

$$
(\alpha \wedge \beta, \gamma)=(\alpha, \beta \wedge \gamma)
$$

Hence if $\mathcal{A}$ has a nice integral, $(H, \wedge,(\cdot, \cdot))$ is a Frobenius algebra.

1.4. Formal Frobenius supermanifolds from DGBV algebras. The construction of Frobenius manifold structure is based on the existence of a solution $\Gamma=\sum \Gamma_{n}$ to

$$
\begin{gathered}
\delta \Gamma+\frac{1}{2}[\Gamma \bullet \Gamma]=0, \\
\Delta \Gamma=0,
\end{gathered}
$$

which satisfies the following conditions: (a) $\Gamma_{0}=0$; (b) $\Gamma_{1}=\sum x^{j} e_{j}, e_{j} \in$ $\operatorname{Ker} \delta \cap \operatorname{Ker} \Delta$, where the classes of $e_{j}$ 's generate $H=H(\mathcal{A}, \delta) ;(\mathrm{c})$ for $n>1$, $\Gamma_{n} \in \operatorname{Im} \Delta$ is a homogeneous super polynomial of degree $n$ in $x^{j}$ 's, such that the total degree of $\Gamma_{n}$ is even; (d) $x^{0}$ only appears in $\Gamma_{1}$. Such a solution is called a normalized universal solution. Under suitable conditions, its existence can be established inductively. This is how Tian [23] and Todorov [24] proved that the deformation of complex structures on a Calabi-Yau manifold is unobstructed. It was later generalized by Bershadsky-Cecotti-Ooguri-Vafa [2] to the case of extended moduli space of complex structures of a Calabi-Yau manifold. Barannikov and Kontsevich [1] used the above results to construct formal Frobenius manifold structures. They also remarked that similar construction can be carried out for DGBV algebras with suitable conditions. The detailed exposition was given in Manin [17]. Their result is summarized as the following

Theorem 1.1. Let $(\mathcal{A}, \wedge, \delta, \Delta,[\cdot \bullet \cdot])$ be a $D G B V$ algebra satisfying the following conditions:

(1) $H=H(\mathcal{A}, \delta)$ is finite dimensional.

(2) There is a nice integral on $\mathcal{A}$.

(3) The inclusions $(\operatorname{Ker} \Delta, \delta) \hookrightarrow(\mathcal{A}, \delta)$ and $(\operatorname{Ker} \delta, \Delta) \hookrightarrow(\mathcal{A}, \Delta)$ induce isomorphisms of cohomology.

Then there is a formal Frobenius manifold structure on $H$. 
1.5. Potential function. The potential function of the formal Frobenius manifold structure in Theorem 1.1 was explicitly given in [1] and [17]: let $\Gamma=$ $\Gamma_{1}+\Delta B$ be a normalized solution, then

$$
\Phi=\int \frac{1}{6} \Gamma^{3}-\frac{1}{2} \delta B \Delta B .
$$

Note that this is the action given in Bershadsky et al [2] in different notations. Following Manin [17], we shall rewrite the second term in a different form so that the potential function is clearly seen to depend only on the normalized solution $\Gamma$. This turns out to be very useful in the identification. Since $\Delta \Gamma=0$, we have

$$
[\Gamma \bullet \Gamma]=\Delta(\Gamma \wedge \Gamma)-(\Delta \Gamma) \wedge \Gamma-\Gamma \wedge(\Delta \Gamma)=\Delta(\Gamma \wedge \Gamma) .
$$

Hence,

$$
\begin{aligned}
& \int \delta B \wedge \Delta B=\int \Delta \delta B \wedge B=-\int \delta \Delta B \wedge B \\
= & -\int \delta\left(\Gamma-\Gamma_{1}\right) \wedge B=-\int \delta \Gamma \wedge B=\int \frac{1}{2}[\Gamma \bullet \Gamma] \wedge B \\
= & \frac{1}{2} \int \Delta(\Gamma \wedge \Gamma) \wedge B=\frac{1}{2} \int \Gamma \wedge \Gamma \wedge \Delta B .
\end{aligned}
$$

So we have

$$
\Phi=\int \frac{1}{6} \Gamma^{3}-\frac{1}{4} \Gamma \wedge \Gamma \wedge \Delta B=\int \frac{1}{6} \Gamma^{3}-\frac{1}{4} \Gamma \wedge \Gamma \wedge\left(\Gamma-\Gamma_{1}\right) .
$$

\section{Formal Frobenius manifold structure on de Rham cohomology}

2.1. DGBV algebra in Poisson geometry. Let $w \in \Gamma\left(X, \Lambda^{2} T X\right)$ be a bi-vector field. It is called a Poisson bi-vector if the Schouten-Nijenhuis bracket $[w, w]$ of $w$ vanishes $[26]$. Let $\mathcal{A}=\Omega(X)$ with the ordinary wedge product $\wedge$, and the exterior differential $d$. Following Koszul [13], we consider $\Delta: \Omega^{*}(X) \rightarrow$ $\Omega^{*-1}(X)$ defined by $\Delta \alpha=w \vdash d \alpha-d(w \vdash \alpha)$, for $\alpha \in \Omega^{*}(X)$, where $\vdash$ is the contraction. Koszul [13] proved that $\Delta^{2}=0$ and $d \Delta+\Delta d=0$. He also defined the following bracket:

$$
[\alpha, \beta]_{\Delta}=(-1)^{|\alpha|}\left(\Delta(\alpha \wedge \beta)-(\Delta \alpha) \wedge \beta-(-1)^{|\alpha|} \alpha \wedge(\Delta \beta)\right),
$$

$\alpha, \beta \in \Omega(X)$, and showed that $(\mathcal{A}, \wedge, \delta=d, \Delta,[\cdot \bullet \cdot])$ is a DGBV algebra, even though he did not explicitly use the term DGBV algebras. For example, the recognization of DGBV algebras in Poisson geometry can be found in $\mathrm{Xu}$ [28].

When $X$ is closed and oriented, let $\int: \mathcal{A} \rightarrow \mathbb{R}$ be the ordinary integral of differential forms over $X$. Then clearly (1) is satisfied. To check (2), we need the following

Lemma 2.1. If $\alpha, \beta \in \Omega^{*}(X)$ satisfy $|\alpha|+|\beta|=\operatorname{dim}(X)+2$, then we have

$$
\int_{X}(w \vdash \alpha) \wedge \beta=\int_{X} \alpha \wedge(w \vdash \beta) .
$$


Proof. Without loss of generality, we assume that the bi-vector field $w=$ $w^{i j} e_{i} \wedge e_{j}$ for some vector fields $e_{i}$ over $X$, where $w^{i j}$ are smooth functions on $X$. (Indeed, we use a partition of unity to decompose $w$ into a sum of bi-vector fields which can be written this way.) Notice that $\left(e_{j} \vdash \alpha\right) \wedge \beta$ and $\alpha \wedge\left(e_{i} \vdash \beta\right)$ have degree $|\alpha|+|\beta|-1=\operatorname{dim}(X)+1$, hence they must vanish. Then we have

$$
\begin{aligned}
& \int_{X}(w \vdash \alpha) \wedge \beta=\int_{X} w^{i j}\left(e_{i} \vdash e_{j} \vdash \alpha\right) \wedge \beta \\
= & \int_{X} w^{i j} e_{i} \vdash\left[\left(e_{j} \vdash \alpha\right) \wedge \beta\right]-\int_{X} w^{i j}(-1)^{|\alpha|-1}\left(e_{j} \vdash \alpha\right) \wedge\left(e_{i} \vdash \beta\right) \\
= & (-1)^{|\alpha|} \int_{X} w^{i j} e_{j} \vdash\left[\alpha \wedge\left(e_{i} \vdash \beta\right)\right]-w^{i j}(-1)^{|\alpha|} \alpha \wedge\left(e_{j} \vdash e_{i} \vdash \beta\right) \\
= & -\int_{X} w^{i j} \alpha \wedge\left(e_{j} \vdash e_{i} \vdash \beta\right)=\int_{X} \alpha \wedge(w \vdash \beta) .
\end{aligned}
$$

Proposition 2.1. For any bi-vector field on a closed oriented $X$, we have

$$
\int_{X}(\Delta \alpha) \wedge \beta=(-1)^{|\alpha|} \int_{X} \alpha \wedge \Delta \beta
$$

Proof. Using $\Delta \alpha=w \vdash d \alpha-d(w \vdash \alpha)$, Lemma 2.1 and Stokes theorem, we have

$$
\begin{aligned}
& \int_{X}(\Delta \alpha) \wedge \beta=\int_{X}(w \vdash d \alpha) \wedge \beta-\int_{X} d(w \vdash \alpha) \wedge \beta \\
= & \int_{X} d \alpha \wedge(w \vdash \beta)+(-1)^{|\alpha|} \int_{X}(w \vdash \alpha) \wedge d \beta \\
= & (-1)^{|\alpha|+1} \int_{X} \alpha \wedge d(w \vdash \beta)+(-1)^{|\alpha|} \int_{X} \alpha \wedge(w \vdash d \beta) \\
= & (-1)^{|\alpha|} \int_{X} \alpha \wedge \Delta \beta .
\end{aligned}
$$

By Poincaré duality, $\int$ induces a nondegenerate pairing on $H=H(\mathcal{A}, d)$, which is the de Rham cohomology. Since $X$ is compact, $H$ is finite dimensional. Hence only Condition 3 in Theorem 1.1 remains to satisfy. Thus, we have

Theorem 2.1. Assume that $(X, w)$ is a closed oriented Poisson manifold such that the inclusions $i:(\operatorname{Ker} \Delta, d) \hookrightarrow(\Omega(X), d)$ and $j:(\operatorname{Ker} d, \Delta) \hookrightarrow(\Omega(X), \Delta)$ induce isomorphisms $H(i)$ and $H(j)$ on cohomologies respectively. Then there is a structure of formal Frobenius manifold on $H^{*}(X)$.

For a symplectic manifold $\left(X^{2 n}, \omega\right)$, Brylinski [4] defined the symplectic star operator $*_{\omega}: \Omega^{k}(X) \rightarrow \Omega^{2 n-k}(X)$. He showed that $\Delta=(-1)^{k} *_{\omega} d *_{\omega}$ on $\Omega^{k}(X)$ (Brylinski [4], Theorem 2.2.1, where $\delta$ is used instead of $\Delta$ ). From this it is clear that $H(i)$ is an isomorphism if and only if $H(j)$ is an isomorphism. Hence it suffices to consider $H(i)$.

On a symplectic manifold $\left(X^{2 n}, \omega\right)$, notice that the inclusion $\phi: \operatorname{Ker} d \cap$ $\operatorname{Ker} \Delta \rightarrow(\Omega(X), d)$ factors through the inclusions $\psi: \operatorname{Ker} d \cap \operatorname{Ker} \Delta \rightarrow(\operatorname{Ker} \Delta, d)$ 
and $i:(\operatorname{Ker} \Delta, d) \rightarrow(\Omega(X), d)$. It is easy to see that $H(\psi)$ is surjective with kernel $d \operatorname{Ker} \Delta$. Therefore, we have an isomorphism

$$
\tilde{H}(\psi): \operatorname{Ker} d \cap \operatorname{Ker} \Delta / d \operatorname{Ker} \Delta \cong H(\operatorname{Ker} \Delta, d) .
$$

Similarly, the kernel of $H(\phi)$ is $\operatorname{Im} d \cap \operatorname{Ker} \Delta$, so we have an injective homomorphism

$$
\tilde{H}(\phi): \operatorname{Ker} d \cap \operatorname{Ker} \Delta / \operatorname{Im} d \cap \operatorname{Ker} \Delta \rightarrow H(X) .
$$

Also since $d \operatorname{Ker} \Delta \subset \operatorname{Im} d \cap \operatorname{Ker} \Delta$, we have a surjective homomorphism $q$ : $\operatorname{Ker} d \cap \operatorname{Ker} \Delta / d \operatorname{Ker} \Delta \rightarrow \operatorname{Ker} d \cap \operatorname{Ker} \Delta / \operatorname{Im} d \cap \operatorname{Ker} \Delta$. To summarize, we get a commutative diagram

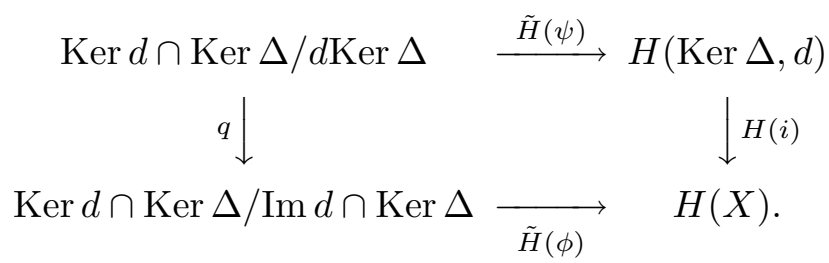

It is then clear that $H(i)$ is injective if and only if $d \operatorname{Ker} \Delta=\operatorname{Im} d \cap \operatorname{Ker} \Delta$ (see also Manin [17], (5.14)). On the other hand, $H(i)=\tilde{H}(\phi) q \tilde{H}(\psi)^{-1}$, from which we see that $H(i)$ is surjective if and only if $\tilde{H}(\phi)$ is surjective. The problem of surjectivity of $\tilde{H}(\phi)$ is equivalent to the following question asked by Brylinski [4]: whether every class in $H(X)$ can be represented by an element in $\operatorname{Ker} d \cap \operatorname{Ker} \Delta$. He answered this question affirmatively for Käher manifolds. For general symplectic manifolds, Mathieu [18] and Yan [29] proved the following result by different methods:

Proposition 2.2. For any symplectic manifold $\left(X^{2 n}, \omega\right)$, not necessarily compact, the following two statements are equivalent:

(a). $\tilde{H}(\phi)$ is surjective.

(b). For each $0 \leq k \leq n, L^{k}: H^{n-k}(X) \rightarrow H^{n+k}(X)$ is surjective, where $L$ is induced by wedge product with $\omega$.

For closed symplectic manifolds, Mathieu [18] observed that (b) is equivalent to each $L^{k}$ being an isomorphism on $H^{n-k}(X)$ because $H^{n-k}(X)$ and $H^{n+k}(X)$ have the same dimension. If (b) holds, one says that $(X, \omega)$ satisfies the hard Lefschetz theorem. So $H(i)$ is surjective if and only if the symplectic manifold $(X, \omega)$ satisfies the hard Lefschetz theorem. One can find examples which do not satisfy the hard Lefschetz theorem in Mathieu's paper. So not every closed symplectic manifold satisfies the conditions in Theorem 2.1. Nevertheless, we will show that closed Kähler manifolds do satisfy these conditions.

2.2. De Rham Frobenius manifolds for Kähler manifolds. In CaoZhou [6], we constructed formal Frobenius manifold structure on the Dolbeault cohomology for Kähler manifolds by DGBV algebra method. Here we carry out a similar construction on the de Rham cohomology.

For a Kähler manifold $(X, \omega)$, let $L(\alpha)=\omega \wedge \alpha$, and $\Lambda$ be the adjoint of the operator $L$ defined by the Hermitian metric. Then we have

$$
\Delta=\Lambda d-\Lambda d=[\Lambda, d]
$$


From the Hodge identities (Griffith-Harris [11], p. 111), we get

$$
\Delta=-4 \pi\left(d^{c}\right)^{*}=\sqrt{-1}\left(\bar{\partial}^{*}-\partial^{*}\right) .
$$

Where

$$
d^{c}=\frac{\sqrt{-1}}{4 \pi}(\bar{\partial}-\partial)
$$

It is clear that $\Delta^{*}=-4 \pi d^{c}=-\sqrt{-1}(\bar{\partial}-\partial)$. Let $\square_{\Delta}=\Delta \Delta^{*}+\Delta^{*} \Delta$.

Lemma 2.2. We have $\square_{\Delta}=\square$, where $\square=d d^{*}+d^{*} d$ is the Hodge Laplacian.

Proof. An easy calculation gives

$$
\begin{aligned}
& \Delta^{*} \Delta=\partial \partial^{*}+\bar{\partial} \bar{\partial}^{*}-\bar{\partial} \partial^{*}-\partial \bar{\partial}^{*} \\
& \Delta^{*}=\partial^{*} \partial+\bar{\partial}^{*} \bar{\partial}-\bar{\partial}^{*} \partial-\partial^{*} \bar{\partial}
\end{aligned}
$$

Then we have

$$
\square_{\Delta}=\square_{\partial}+\square_{\bar{\partial}}+\left(\bar{\partial} \partial^{*}+\partial^{*} \bar{\partial}\right)-\left(\partial \bar{\partial}^{*}+\bar{\partial}^{*} \partial\right) .
$$

The lemma follows from the following well-known formulas in Kähler geometry (Griffiths-Harris [11], p. 115):

$$
\begin{gathered}
\square=2 \square_{\partial}=2 \square_{\bar{\partial}}, \\
\bar{\partial} \partial^{*}+\partial^{*} \bar{\partial}=\partial \bar{\partial}^{*}+\bar{\partial}^{*} \partial=0 .
\end{gathered}
$$

Standard Hodge theory argument gives a decomposition

$$
\Omega(X)=\mathcal{H} \oplus \operatorname{Im} \Delta \oplus \operatorname{Im} \Delta^{*},
$$

where $\mathcal{H}$ is the space of harmonic forms on $X$. Furthermore, the inclusion $(\mathcal{H}, 0) \subset(\Omega(X), d)$ induces an isomorphism $H(\Omega(X), \Delta) \cong \mathcal{H}$. Notice that $\Delta$ commutes with $\square_{\Delta}$, so it commutes with $\square$.

Lemma 2.3. On a Kähler manifold, $d \Delta^{*}+\Delta^{*} d=0$ and $\Delta d^{*}+d^{*} \Delta=0$.

Proof. The first identity follows from the following identity

$$
d d^{c}=-d^{c} d=\frac{\sqrt{-1}}{2 \pi} \partial \bar{\partial} .
$$

Taking formal adjoint gives the second identity.

Proposition 2.3. Let $(X, \omega)$ be a closed Kähler manifold, then the inclusions $i:(\operatorname{Ker} \Delta, d) \subset(\Omega(X), d)$ and $j:(\operatorname{Ker} d, \Delta) \subset(\Omega(X), \Delta)$ induce isomorphisms on cohomology. 
Proof. By Hodge theory, we have the following orthogonal decompositions:

$$
\Omega(X)=\mathcal{H} \oplus \operatorname{Im} d \oplus \operatorname{Im} d^{*}=\mathcal{H} \oplus \operatorname{Im} \Delta \oplus \operatorname{Im} \Delta^{*} .
$$

It follows that we have a five-fold decomposition:

$$
\Omega(X)=\mathcal{H} \oplus \operatorname{Im} d \Delta \oplus \operatorname{Im} d^{*} \Delta \oplus \operatorname{Im} d \Delta^{*} \oplus \operatorname{Im} d^{*} \Delta^{*} .
$$

It is then clear that $\operatorname{Ker} \Delta=\mathcal{H} \oplus \operatorname{Im} d \Delta \oplus \operatorname{Im} d^{*} \Delta$, Ker $\left.d\right|_{\operatorname{Ker} \Delta}=\mathcal{H} \oplus \operatorname{Im} d \Delta$ and $\left.\operatorname{Im} d\right|_{\text {Ker } \Delta}=\operatorname{Im} d \Delta$. Therefore, $H(\operatorname{Ker} \Delta, d) \cong \mathcal{H}$. It then follows that $H(i)$ is an isomorphism. Similarly for $H(j)$.

In conclusion, we have

Theorem 2.2. For any closed Kähler manifold $X$, there is a structure of formal Frobenius manifold on $H^{*}(X)$ obtained from Theorem 1.1 for the DGBV algebra $\left(\Omega(X), \wedge, d, \Delta,[\cdot \bullet \cdot]_{\Delta}\right)$.

2.3. Explicit formula for $\Gamma$. For a closed Kähler manifold $X$, by Hodge theory, we can take $e_{j}$ 's to be harmonic. Hence we automatically have $e_{j} \in$ $\operatorname{Ker} d \cap \operatorname{Ker} \Delta$. In general $\Gamma_{n} \in \operatorname{Im} \Delta$ for $n \geq 2$. Since we have

$$
\operatorname{Im} \Delta=\operatorname{Im} d \Delta \oplus \operatorname{Im} d^{*} \Delta,
$$

we will require $\Gamma_{n}$ to be in $\operatorname{Im} d^{*} \Delta$. Then we have the following uniqueness result.

Lemma 2.4. Let $\Gamma=\sum_{n} \Gamma_{n}$ be a normalized universal solution to the MaurerCartan equation

$$
d \Gamma+\frac{1}{2}[\Gamma \bullet \Gamma]_{\Delta}=0,
$$

with $\Gamma_{1}=\sum_{j} x^{j} e_{j}, e_{j}$ harmonic, $\Gamma_{n} \in \operatorname{Im} d^{*} \Delta$, for $n>1$. Then $\Gamma$ satisfies

$$
\Gamma=\Gamma_{1}-\frac{1}{2} G d^{*} \Delta(\Gamma \wedge \Gamma)=\Gamma_{1}-\frac{1}{2} d^{*} G[\Gamma \bullet \Gamma]_{\Delta},
$$

where $G: \Omega(X) \rightarrow \Omega(X)$ is the Green's operator of $\square$. Alternatively, for $n>1$,

$$
\Gamma_{n}=-\frac{1}{2} \sum_{j+k=n} G d^{*}\left[\Gamma_{j} \bullet \Gamma_{k}\right]_{\Delta}=-\frac{1}{2} \sum_{j+k=n} G d^{*} \Delta\left(\Gamma_{j} \wedge \Gamma_{k}\right) .
$$

Proof. This is equivalent to solving the Maurer-Cartan equation inductively by imposing the above conditions. Since $\Delta \Gamma=0,[\Gamma \bullet \Gamma]_{\Delta}=\Delta(\Gamma \wedge \Gamma)$. So we need to solve

$$
d \Gamma=-\frac{1}{2} \Delta(\Gamma \wedge \Gamma)
$$

Take $d^{*}$ on both sides:

$$
d^{*} d \Gamma=-\frac{1}{2} d^{*} \Delta(\Gamma \wedge \Gamma)
$$

Since $d^{*} \Gamma=0$, this is equivalent to

$$
\square \Gamma=-\frac{1}{2} d^{*} \Delta(\Gamma \wedge \Gamma) .
$$


Taking Green's operator on both sides then proves (7). Expanding in power series yields (8).

Conversely, it is straightforward to verify the following:

Lemma 2.5. Let $\Gamma=\sum_{n} \Gamma_{n}$ be a power series with $\Gamma_{1}=\sum_{j} x^{j} e_{j}, e_{j}$ harmonic, and for $n>1$,

$$
\Gamma_{n}=-\frac{1}{2} \sum_{j+k=n} G d^{*} \Delta\left(\Gamma_{j} \wedge \Gamma_{k}\right) .
$$

Then $\Gamma$ is a solution to the Maurer-Cartan equation (6).

We call a solution as in Lemma 2.4 analytically normalized. Restricting an analytically normalized solution to $H^{\text {even }}$, we get a power series on $H^{\text {even }}$. Now by modifying a standard argument in Kodaira-Spencer-Kuranishi deformation theory (see e.g. Morrow-Kodaira [19]. Chapter 4, Proposition 2.4), this series has a positive convergent radius. This method was also used by Tian [23] and Todorov [24].

\section{Identification with formal Frobenius supermanifold on Dolbeault cohomology}

By Hodge theory, one can identify the Dolbeault cohomology of a closed Kähler manifold with its complexified de Rham cohomology. In this section, we identify the formal Frobenius manifold constructed in Theorem 2.2 with the one we constructed on the Dolbeault cohomology in [6].

3.1. Formal Frobenius manifold structure on Dolbeault cohomology. We review the construction of Cao-Zhou [6] in this section. Let $(X, g, J)$ be a closed Kähler manifold with Kähler form $\omega$. We need a slight modification. Consider the quadruple $\left(\Omega^{*, *}(X), \wedge, \delta=\bar{\partial}, \Delta=-\sqrt{-1} \partial^{*}\right)$. It is well-known that $\bar{\partial}^{2}=0,\left(\partial^{*}\right)^{2}=0$, and $\bar{\partial} \partial^{*}+\partial^{*} \bar{\partial}=0$. Also, $\bar{\partial}$ is a derivation. Set

$$
[a \bullet b]_{-\sqrt{-1} \partial^{*}}=-\sqrt{-1}(-1)^{|a|}\left(\partial^{*}(a \wedge b)-\partial^{*} a \wedge b-(-1)^{|a|} a \wedge \partial^{*} b\right) .
$$

It was proved in [6] that $\left(\Omega^{*, *}(X), \wedge, \delta=\bar{\partial}, \Delta=-\sqrt{-1} \partial^{*},[\cdot \bullet \cdot]_{-\sqrt{-1} \partial^{*}}\right)$ is a DGBV algebra. Furthermore, let $\int_{X}: \Omega^{*, *}(X) \rightarrow \mathbb{C}$ be the ordinary integration of differential forms. Then $\int_{X}$ is a nice integral for the above DGBV algebra. Hodge theoretical argument similar to the one in last section shows that the two natural inclusions $i:\left(\operatorname{Ker} \partial^{*}, \bar{\partial}\right) \rightarrow\left(\Omega^{*}, *(X), \bar{\partial}\right)$ and $j:\left(\operatorname{Ker} \bar{\partial}, \partial^{*}\right) \rightarrow$ $\left(\Omega^{* * *}(X), \partial^{*}\right)$ induce isomorphisms on cohomology. Therefore, Theorem 1.1 applies to give a formal Frobenius manifold structure on the Dolbeault cohomology.

Remark 3.1. Similarly, set

$$
[a \bullet b]_{\sqrt{-1} \bar{\partial}^{*}}=\sqrt{-1}(-1)^{|a|}\left(\bar{\partial}^{*}(a \wedge b)-\bar{\partial}^{*} a \wedge b-(-1)^{|a|} a \wedge \bar{\partial}^{*} b\right) .
$$

Then $\left(\Omega^{*, *}(X), \wedge, \delta=\partial, \Delta=\sqrt{-1} \bar{\partial}^{*},[\cdot \bullet \cdot]_{\sqrt{-1}} \bar{\partial}^{*}\right)$ is also a DGBV algebra. 
3.2. The identification. By Hodge theory, there is a natural isomorphism between $H\left(\Omega^{*, *}(X), \bar{\partial}\right)$ and $\mathcal{H}_{\bar{\partial}}$, the space of $\bar{\partial}$-harmonic forms. Now $\square_{\bar{\partial}}=\frac{1}{2} \square$, the space of $\square$-harmonic forms is the same as the space of $\square \square_{\bar{\partial}}$-forms. Let $\Gamma=$ $\sum_{n} \Gamma_{n}$ be a normalized solution to

$$
\bar{\partial} \Gamma+\frac{1}{2}[\Gamma \bullet \Gamma]_{-\sqrt{-1} \partial^{*}}=0,
$$

such that $\Gamma_{1}=\sum_{j} x^{j} e_{j}, e_{j} \bar{\partial}$-harmonic, and $\Gamma_{n} \in \operatorname{Im} \bar{\partial}^{*} \partial^{*}$, for $n>1$. By the method of $\S 2.3$, we have

$$
\Gamma_{n}=\frac{1}{2} \sqrt{-1} \sum_{j+k=n} G_{\bar{\partial}} \bar{\partial}^{*} \partial^{*}\left(\Gamma_{j} \wedge \Gamma_{k}\right) .
$$

Lemma 3.1. We have $G d^{*} \Delta=-\sqrt{-1} G_{\bar{\partial}} \bar{\partial}^{*} \partial^{*}$.

Proof. This follows from $d=\partial+\bar{\partial}, \Delta=\sqrt{-1}\left(\bar{\partial}^{*}-\partial^{*}\right)$ and $G=\frac{1}{2} G_{\bar{\partial}}$.

As a corollary, we see that the Maurer-Cartan equations (6) and (9) share the same analytically normalized solutions. By the explicit formula (3) for the potential function, we have

Theorem 3.1. For any closed Kähler manifold $X$, the formal Frobenius manifold structure on Dolbeault cohomology in $\S 3.1$ can be identified with the formal Frobenius manifold structure on complexified de Rham cohomology in $§ 2.2$.

\section{Acknowledgements}

The authors would like to thank Gang Tian and S.-T. Yau for their interest. The work on this paper was carried out while the second author was visiting Texas A\&M University. He would like to express his appreciation for the hospitality and financial support of the Mathematics Department and the GeometryAnalysis-Topology group.

\section{References}

[1] S. Barannikov and M. Kontsevich, Frobenius manifolds and formality of lie algebras of polyvector fields, preprint, alg-geom/9710032.

[2] M. Bershadsky, S. Cecotti, H. Ooguri, and C. Vafa, Kodaira-Spencer theory of gravity and exact results for quantum string amplitudes Comm. Math. Phys. 165 (1994), 311-427.

[3] M. Bershadsky, V. Sadov, Theory of Kähler gravity Internat. J. Modern Phys. A 11 (1996),4689-4730.

[4] J.-L. Brylinski, A differential complex for Poisson manifolds, J. Differential Geom. 28 (1988),93-114.

[5] P. Candelas, X.C. de la Ossa, p.S. Green, and L. Parkes, A pair of Calabi-Yau manifolds as an exactly soluble superconformal theory, Nuclear Phys. B 359 (1991), 21-74.

[6] H.-D. Cao and J. Zhou, Frobenius manifold structure on Dolbeault cohomology and mirror symmetry, preprint, May, 1998.

[7] B. Dubrovin, Integrable systems in topological field theory, Nuclear Phys. B 379 (1992), 627-689.

[8] - Geometry of 2D topological field theories, in Integrable systems and quantum groups (Montecatini Terme, 1993), 120-348, Lecture Notes in Math., 1620, Springer, Berlin, 1996. 
[9] M. Gerstenhaber and S.D. Schack, Algebraic cohomology and deformation theory, in em Deformation theory of algebras and structures and applications (Il Ciocco, 1986), 11-264, NATO Adv. Sci. Inst. Ser. C: Math. Phys. Sci., 247, Kluwer Acad. Publ., Dordrecht, 1988.

[10] B.R. Greene and M.R. Plesser, Duality in Calabi-Yau moduli space, Nuclear Phys. B338 (1990), 15-37.

[11] P. Griffiths and J. Harris, Principles of algebraic geometry. Pure and Applied Mathematics. Wiley-Interscience [John Wiley \& Sons], New York, 1978.

[12] M. Kontsevich, Deformation quantization of Poisson manifolds, I, preprint, q$\operatorname{alg} / 9709040$.

[13] J.-L. Koszul, Crochet de Schouten-Nijenhuis et cohomologie, The mathematical heritage of Élie Cartan (Lyon, 1984). Astérisque 1985, Numero Hors Serie, 257-271.

[14] J. Li and G. Tian, Comparison of the algebraic and the symplectic Gromov-Witten invariants, preprint, alg-geom/9712035.

[15] B. Lian, K. Liu, and S.-T. Yau, Mirror principle I, preprint, alg-geom/9712011.

[16] Y. Manin, Frobenius manifolds, quantum cohomology, and moduli spaces (Chapters I, II, III). preprint, MPI 96-113, 1996.

[17] , Three constructions of Frobenius manifolds: a comparative study, preprint, math.QA/9801006.

[18] O. Mathieu, Harmonic cohomology classes of symplectic manifolds, Comment. Math. Helv. 70 (1995), 1-9.

[19] J. Morrow and K. Kodaira, Complex manifolds, Holt, Rinehart and Winston, Inc., New York-Montreal, Que.-London, 1971.

[20] Z. Ran, Thickening Calabi-Yau moduli spaces, in Mirror symmetry, II, 393-400, AMS/IP Stud. Adv. Math., 1, Amer. Math. Soc., Providence, RI, 1997.

[21] Y. Ruan and G. Tian, A mathematical theory of quantum cohomology, J. Differential Geom. 42 (1995), 259-367.

[22] A. Strominger, S.-T. Yau, and E. Zaslow, Mirror symmetry is T-duality, Nuclear Phys. B 479 (1996), 243-259.

[23] G. Tian, Smoothness of the universal deformation space of compact Calabi-Yau manifolds and its Petersson-Weil metric, in Mathematical aspects of string theory (San Diego, Calif., 1986), 629-646, Adv. Ser. Math. Phys., 1, World Sci. Publishing, Singapore, 1987.

[24] A.N. Todorov, The Weil-Petersson geometry of the moduli space of $S U(n \geq 3)$ (CalabiYau) manifolds. I., Comm. Math. Phys. 126 (1989), 325-346.

[25] C. Vafa, Topological mirrors and quantum rings, in Essays on mirror manifolds, S.-T. Yau ed., 96-119, Internat. Press, Hong Kong, 1992.

[26] I. Vaisman, Lectures on the geometry of Poisson manifolds. Progress in Mathematics, 118. Birkhäuser Verlag, Basel, 1994.

[27] E. Witten, Mirror manifolds and topological field theory, in Essays on mirror manifolds, S. T. Yau ed., International Press, Hong Kong, 1992.

[28] P. Xu, Gerstenhaber algebras and BV-algebras in Poisson geometry, preprint, dgga/9703001.

[29] D. Yan, Hodge structure on symplectic manifolds, Adv. Math. 120 (1996), 143-154.

[30] S.-T. Yau ed., Essays on mirror manifolds, International Press, Hong Kong, 1992.

Department of Mathematics, Texas A \& M University, College Station, TX 77843

E-mail address: cao@math.tamu.edu, zhou@math.tamu.edu 